\title{
Quantum polarization distributions via marginals of quadrature distributions
}

\author{
Alfredo Luis* \\ Departamento de Óptica, Facultad de Ciencias Físicas, Universidad Complutense, 28040 Madrid, Spain
}

(Received 14 November 2004; published 2 May 2005)

\begin{abstract}
We derive quantum polarization distributions on the Poincaré sphere for two-mode fields as suitable marginals of the standard $s$-ordered quadrature distributions that include the Wigner function. We compare them with previously introduced distributions directly defined on the sphere from first principles. We apply this approach to some relevant field states such as coherent and number states.
\end{abstract}

DOI: 10.1103/PhysRevA.71.053801

PACS number(s): 42.50.Dv, 03.65.Ca

\section{INTRODUCTION}

Polarization is a crucial ingredient of light in both the classical and quantum domains. However, quantum polarization is mainly addressed in terms of the abstract Hilbertspace logic in a finite-dimensional space. This is rather divorced from the usual language used in the classical domain in terms of the Stokes parameters and the Poincaré sphere. Because of this, we think that it is worth developing and investigating formulations of quantum polarization as distributions on the Poincaré sphere. Such approaches are closer to our common intuition about polarization phenomena, so they can be very helpful in understanding quantum polarization properties.

In this regard we can follow two different routes. On the one hand, we have a direct approach by translating to polarization previously introduced phase-space formalisms for angular momentum and spin variables [1-7]. This is possible because the Stokes parameters (the basic variables describing polarization) are formally equivalent to an angular momentum. For definiteness we focus on the proposals in Refs. [1-5] referring to them as $\mathrm{SU}(2)$ distributions.

On the other hand, we can derive polarization distributions via suitable marginals of distributions for the complex amplitudes (or field quadratures) by removing the degrees of freedom irrelevant for the specification of polarization. This is the approach investigated in this work, and we refer to them as marginal distributions. More specifically, we consider proper marginals of $s$-ordered quadrature distributions that include distinguished particular examples such as the $Q$, $P$, and Wigner functions [8].

There are several reasons supporting the expediency of this approach to quantum polarization. From a practical perspective, we have that polarization distributions provide a feasible approach to examine and measure diverse polarization properties. This is the case of the degree of polarization recently introduced as the distance between the polarization distribution and the uniform distribution associated with unpolarized light [9]. This can be also the case of the proper assessment of polarization correlations derived from the properties of the joint polarization distribution [10]. Polariza-

*Electronic address: alluis@ fis.ucm.es; URL: http://www.ucm.es/ info/gioq tion correlations are crucial for recently developed applications of quantum theory [11]. Marginals of suitable quadrature distributions have been used also to study phase properties of one- and two-mode quantum field states [12].

In this regard we may say that marginal distributions provide the most down-to-earth approach to quantum polarization distributions, since, by definition, they are to be obtained exactly in the same way as polarization distributions are derived in classical optics [13]. This is in sharp contrast to SU(2) distributions whose definitions bear no definite relation with optics, being introduced specifically for the description of abstract angular momenta.

Among the large family of quantum phase-space distributions known in physics, the $s$-ordered distributions are distinguished by their good theoretical and experimental properties. For example, in the context of polarization we have that $s$-ordered quadrature distributions transform properly under the transformations that represent action of standard polarization changing devices [14].

From the experimental perspective, s-ordered distributions can be determined in practice by using diverse experimental procedures, such as homodyne and heterodyne detection, tomography, and atom-field interactions, to mention just the most popular and repeatedly carried out experimentally [15]. As a matter of fact, most of these practical schemes are very simple so that their operation can be understood even within a purely classical framework. Moreover, they are robust against experimental imperfections, such as detection inefficiencies, that imply just a change of the value of the $s$ parameter.

This widespread measurability is not matched by any other family of phase-space distributions. This is also in sharp contrast to the case of $\mathrm{SU}(2)$ distributions which, to the best of our knowledge, have not been determined experimentally yet. In any case, the theoretical proposals for their practical determination are rather cumbersome and lack the simple and intuitive picture provided by schemes measuring the $s$-ordered distributions $[3,16]$.

Finally, s-ordered distributions provide a simple measure of the degree of nonclassical behavior of quantum states [17].

In Sec. II we derive the main formulas establishing the operator-function correspondence obtained after removing from the quadrature distributions the variables not related to the polarization. In Secs. III and IV we examine their main properties, comparing the marginal distributions with the 
$\mathrm{SU}(2)$ distributions. In Sec. V we apply this approach to some relevant examples of field states such as coherent and number states. Finally, in Sec. VI we examine whether this approach can be used to measure the degree of polarization of quantum fields.

\section{MARGINAL DISTRIBUTIONS FOR POLARIZATION}

Our starting point is the $s$-ordered distributions $W\left(\alpha_{1}, \alpha_{2} ; s\right)$ for quadrature variables:

$$
W\left(\alpha_{1}, \alpha_{2} ; s\right)=\operatorname{tr}\left[\rho T\left(\alpha_{1} ; s\right) \otimes T\left(\alpha_{2} ; s\right)\right],
$$

where $\rho$ is the two-mode density matrix, $\alpha_{1}$ and $\alpha_{2}$ are the complex amplitudes for each field mode, $T\left(\alpha_{j} ; s\right)$ is the phase-space point operator [8],

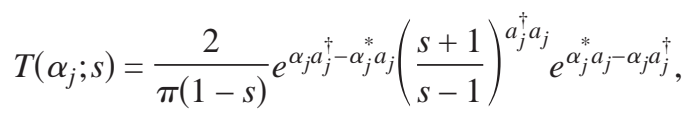

and $a_{j}$ is the complex amplitude operator for the corresponding field mode. This definition is normalized so that

$$
\int d^{2} \alpha_{1} d^{2} \alpha_{2} W\left(\alpha_{1}, \alpha_{2} ; s\right)=1 .
$$

This includes the $Q$ function $(s=-1)$, the $P$ function $(s=1)$, and the Wigner function $(s=0)$.

In order to remove the variables not entering into the idea of polarization (i.e., total intensity and global phase) we perform the change of variables

$$
\alpha_{1}=r \cos \frac{\theta}{2} e^{i \delta}, \quad \alpha_{2}=r \sin \frac{\theta}{2} e^{i \delta} e^{i \phi},
$$

with

$$
d^{2} \alpha_{1} d^{2} \alpha_{2}=\frac{1}{4} r^{3} d r d \delta \sin \theta d \theta d \phi .
$$

In this parametrization $r^{2}=\left|\alpha_{1}\right|^{2}+\left|\alpha_{2}\right|^{2}$ represents the total intensity, $\delta$ is a global phase, $\phi=\arg \alpha_{2}-\arg \alpha_{1}$ is the phase difference, and $\tan (\theta / 2)=\left|\alpha_{2}\right| /\left|\alpha_{1}\right|$ describes the relative amount of intensity carried by each field mode.

The two variables $\Omega=(\theta, \phi)$ are the parameters defining a polarization state, which can be represented as a point on a unit sphere: the Poincaré sphere. Our first objective is to derive from $T\left(\alpha_{1} ; s\right) \otimes T\left(\alpha_{2} ; s\right)$ a family of phase-space point operators $\Delta(\Omega ; s)$ for polarization by removing the other degrees of freedom (i.e., $r$ and $\delta$ ) irrelevant for the specification of polarization state:

$$
\Delta(\Omega ; s)=\frac{1}{4} \int_{0}^{\infty} d r r^{3} \int_{0}^{2 \pi} d \delta T\left(\alpha_{1} ; s\right) \otimes T\left(\alpha_{2} ; s\right) .
$$

This calculation simplifies if we use the good transformation properties of $W(\boldsymbol{\alpha} ; s)$ under $\mathrm{SU}(2)$ transformations (rotations on the Poincaré sphere) [14],

$$
W_{U \rho U^{\dagger}}(\boldsymbol{\alpha} ; s)=W_{\rho}\left(\mathcal{U}^{\dagger} \boldsymbol{\alpha} ; s\right),
$$

where the subscript in $W$ indicates the corresponding operator, $U$ is the unitary operator representing the $\mathrm{SU}(2)$ transformation,

$$
U^{\dagger} \boldsymbol{a} U=\mathcal{U} \boldsymbol{a},
$$

$\mathcal{U}$ a unitary $2 \times 2$ complex matrix, and

$$
\boldsymbol{a}=\left(\begin{array}{l}
a_{1} \\
a_{2}
\end{array}\right), \quad \boldsymbol{\alpha}=\left(\begin{array}{l}
\alpha_{1} \\
\alpha_{2}
\end{array}\right) .
$$

Because of this property, we can always express $\Delta(\Omega ; s)$ as

$$
\Delta(\Omega ; s)=U(\Omega) \Delta(\theta=0 ; s) U^{\dagger}(\Omega),
$$

$U(\Omega)$ being the $\mathrm{SU}(2)$ transformation with

$$
\mathcal{U}^{\dagger}=\left(\begin{array}{cc}
\cos \frac{\theta}{2} & \sin \frac{\theta}{2} e^{-i \phi} \\
-\sin \frac{\theta}{2} e^{i \phi} & \cos \frac{\theta}{2}
\end{array}\right),
$$

so that

$$
\mathcal{U}^{\dagger} \boldsymbol{\alpha}=\left(\begin{array}{c}
r e^{i \delta} \\
0
\end{array}\right)
$$

From Eq. (2.10) we can focus without loss of generality on the north pole $\theta=0$ :

$$
\Delta(\theta=0 ; s)=\frac{1}{4} \int_{0}^{\infty} d r r^{3} \int_{0}^{2 \pi} d \delta T\left(\alpha_{1}=r e^{i \delta} ; s\right) \otimes T\left(\alpha_{2}=0 ; s\right) .
$$

This greatly simplifies the problem since now the removal of $r$ and $\delta$ is performed just on the mode $a_{1}$.

This removal is further simplified by evaluating $T\left(\alpha_{1} ; s\right)$ on the quadrature coherent states $|\beta\rangle$, with $a_{1}|\beta\rangle=\beta|\beta\rangle$. In such a case,

$$
\begin{aligned}
\left\langle\beta\left|T\left(\alpha_{1} ; s\right)\right| \beta\right\rangle= & \frac{2}{\pi(1-s)}\left\langle\beta-\alpha_{1}\left|\left(\frac{s+1}{s-1}\right)^{a_{1}^{\ddagger} a_{1}}\right| \beta-\alpha_{1}\right\rangle \\
= & \frac{2}{\pi(1-s)} \exp \left\{-\frac{2}{1-s}\left[r^{2}+|\beta|^{2}\right.\right. \\
& -2 r|\beta| \cos (\delta-\varphi)]\}
\end{aligned}
$$

where $\varphi=\arg \beta$. The $\delta$ integration gives

$$
\begin{aligned}
& \int_{0}^{2 \pi} d \delta\left\langle\beta\left|T\left(\alpha_{1} ; s\right)\right| \beta\right\rangle \\
& \quad=\frac{4}{1-s} \exp \left[-\frac{2}{1-s}\left(r^{2}+|\beta|^{2}\right)\right] I_{0}\left(\frac{4|\beta| r}{1-s}\right) \\
& \quad=\frac{4}{1-s} \exp \left[-\frac{2}{1-s}\left(r^{2}+|\beta|^{2}\right)\right] \sum_{k=0}^{\infty} \frac{1}{k !^{2}}\left(\frac{2 r|\beta|}{1-s}\right)^{2 k},
\end{aligned}
$$

where $I_{0}$ is the modified Bessel function of first kind and zero order. Finally, the $r$ integration gives 


$$
\begin{aligned}
& \frac{1}{4} \int_{0}^{\infty} d r r^{3} \int_{0}^{2 \pi} d \delta\left\langle\beta\left|T\left(\alpha_{1} ; s\right)\right| \beta\right\rangle=\frac{1-s}{8}\left(1+\frac{2}{1-s}|\beta|^{2}\right) \\
& \quad=\frac{1-s}{8}\left\langle\beta\left|\left(1+\frac{2}{1-s} a_{1}^{\dagger} a_{1}\right)\right| \beta\right\rangle .
\end{aligned}
$$

Therefore,

$$
\Delta(\theta=0 ; s)=\frac{1}{4 \pi}\left(\frac{s+1}{s-1}\right)^{a_{2}^{\dagger} a_{2}}\left(1+\frac{2}{1-s} a_{1}^{\dagger} a_{1}\right) .
$$

Finally, since

$$
\begin{aligned}
& U a_{1} U^{\dagger}=\cos \frac{\theta}{2} a_{1}+e^{-i \phi} \sin \frac{\theta}{2} a_{2}, \\
& U a_{2} U^{\dagger}=\cos \frac{\theta}{2} a_{2}-e^{i \phi} \sin \frac{\theta}{2} a_{1},
\end{aligned}
$$

we get from Eq. (2.10) that

$$
\Delta(\Omega ; s)=\frac{1}{4 \pi}\left(\frac{s+1}{s-1}\right)^{\left(S_{0}-\mathbf{\Omega} \cdot S\right) / 2}\left[1+\frac{1}{1-s}\left(S_{0}+\mathbf{\Omega} \cdot \boldsymbol{S}\right)\right],
$$

where $\boldsymbol{\Omega}$ is the three-dimensional real vector,

$$
\mathbf{\Omega}=\left(\begin{array}{c}
\sin \theta \cos \phi \\
\sin \theta \sin \phi \\
\cos \theta
\end{array}\right),
$$

and $S_{0}$ and $S$ are the Stokes operators:

$$
\begin{gathered}
S_{0}=a_{1}^{\dagger} a_{1}+a_{2}^{\dagger} a_{2}, \quad S_{y}=i\left(a_{2}^{\dagger} a_{1}-a_{1}^{\dagger} a_{2}\right), \\
S_{x}=a_{2}^{\dagger} a_{1}+a_{1}^{\dagger} a_{2}, \quad S_{z}=a_{1}^{\dagger} a_{1}-a_{2}^{\dagger} a_{2} .
\end{gathered}
$$

\section{PROPERTIES AND COMPARISON WITH SU(2) DISTRIBUTIONS}

It can be appreciated that the phase-space point operators $\Delta(\Omega ; s)$ in Eq. (2.19) commute with the total photon-number operator $\left[\Delta(\Omega ; s), S_{0}\right]=0$ so that $\Delta(\Omega ; s)$ leave invariant the subspaces with fixed total number of photons $N$. Therefore we have

$$
\Delta(\Omega ; s)=\sum_{N=0}^{\infty} \Delta^{(N)}(\Omega ; s),
$$

where $\Delta^{(N)}(\Omega ; s)$ is the restriction of $\Delta(\Omega ; s)$ to subspaces of fixed total photon number $N$.

The operators $\Delta^{(N)}(\Omega ; s)$ provide a new phase-space formulation of quantum physics on the sphere for finitedimensional systems of arbitrary dimension $N+1$. The finitedimensional spaces are often regarded as describing an arbitrary angular momentum $\mathbf{j}$ with $\mathbf{j}^{2}=j(j+1)$ (in units in which $\hbar=1)$. The approach developed above can be recast in this language simply by performing the replacements $j$ $=S_{0} / 2$ and $\mathbf{j}=\mathbf{S} / 2$, along with the following relation between number states $\left|n_{1}\right\rangle_{1}\left|n_{2}\right\rangle_{2}$ and the eigenstates $|j, m\rangle$ of $\mathbf{j}^{2}$ and $j_{z}$ :

$$
|j, m\rangle=\left|n_{1}=j+m\right\rangle_{1} \otimes\left|n_{2}=j-m\right\rangle_{2} .
$$

The phase-space approach provided by $\Delta^{(N)}(\Omega ; s)$ does not coincide in general with previously introduced phasespace formalisms for finite-dimensional systems. More specifically, we focus on the angular analogs of the quadrature $s$-ordered distributions,

$$
\mathcal{W}(\Omega ; s)=\operatorname{tr}\left[\rho \Lambda^{(j)}(\Omega ; s)\right],
$$

with $[1-3]$

$$
\begin{aligned}
\Lambda^{(j)}(\Omega ; s)= & \frac{1}{\sqrt{4 \pi}} \sum_{\ell=0}^{2 j} \sum_{m=-\ell}^{\ell} \sum_{k, q=-j}^{j} \sqrt{2 \ell+1} \frac{\langle j, k ; \ell, m \mid j, q\rangle}{\langle j, j ; \ell, 0 \mid j, j\rangle^{s}} \\
& \times Y_{\ell, m}(\Omega)|j, k\rangle\langle j, q|,
\end{aligned}
$$

where $\left\langle j_{1}, m_{1} ; j_{2}, m_{2} \mid j, m\right\rangle$ are the Clebsch-Gordan coefficients and $Y_{\ell, m}(\Omega)$ the spherical harmonics. This definition is normalized so that

$$
\int d \Omega \mathcal{W}(\Omega ; s)=\operatorname{tr} \rho,
$$

with $d \Omega=\sin \theta d \theta d \phi$, as usual. We refer to $\mathcal{W}(\Omega ; s)$ as $\mathrm{SU}(2)$ distributions. Other phase-space approaches for finitedimensional systems can be found in Refs. [6,7].

The marginal distributions (2.19) and the $\mathrm{SU}(2)$ distributions (3.4) only coincide for $s=-1$, since, in such a case,

$$
\Lambda^{(j)}(0 ;-1)=\Delta^{(2 j)}(0 ;-1)=\frac{2 j+1}{4 \pi}|j, j\rangle\langle j, j|,
$$

so that $\Lambda^{(j)}(\Omega ;-1)=\Delta^{(2 j)}(\Omega ;-1)$ are given by projection on the $\mathrm{SU}(2)$ coherent states [5]. This is the definition of the $\mathrm{SU}(2) Q$ function $[5,9,10]$.

For $s \neq-1$ the discrepancy between $\Delta^{(N)}(\Omega ; s)$ and $\Lambda^{(j)}(\Omega ; s)$ is clearly revealed when comparing their spectra. The spectra can be easily computed for $\theta=0$ since in such a case both $\Delta^{(N)}(0 ; s)$ and $\Lambda^{(j)}(0 ; s)$ are diagonal in the $|j, m\rangle$ basis,

$$
\begin{gathered}
\Delta^{(2 j)}(0 ; s)=\sum_{m=-j}^{j} \lambda_{m}|j, m\rangle\langle j, m|, \\
\Lambda^{(j)}=\sum_{m=-j}^{j} \tilde{\lambda_{m}}|j, m\rangle\langle j, m|,
\end{gathered}
$$

being

$$
\begin{aligned}
& \lambda_{m}=\frac{1}{4 \pi}\left(1+2 \frac{j+m}{1-s}\right)\left(\frac{s+1}{s-1}\right)^{j-m}, \\
& \tilde{\lambda}_{m}=\frac{1}{4 \pi} \sum_{\ell=0}^{2 j}(2 \ell+1) \frac{\langle j, m ; \ell, 0 \mid j, m\rangle}{\langle j, j ; \ell, 0 \mid j, j\rangle^{s}} .
\end{aligned}
$$

In Fig. 1 we represent the spectra for $2 j=N=40$ and $s$ $=0, \pm 0.05$. Note that they tend to be rather similar for $s<0$ while they become very dissimilar for $s \geqslant 0$. 

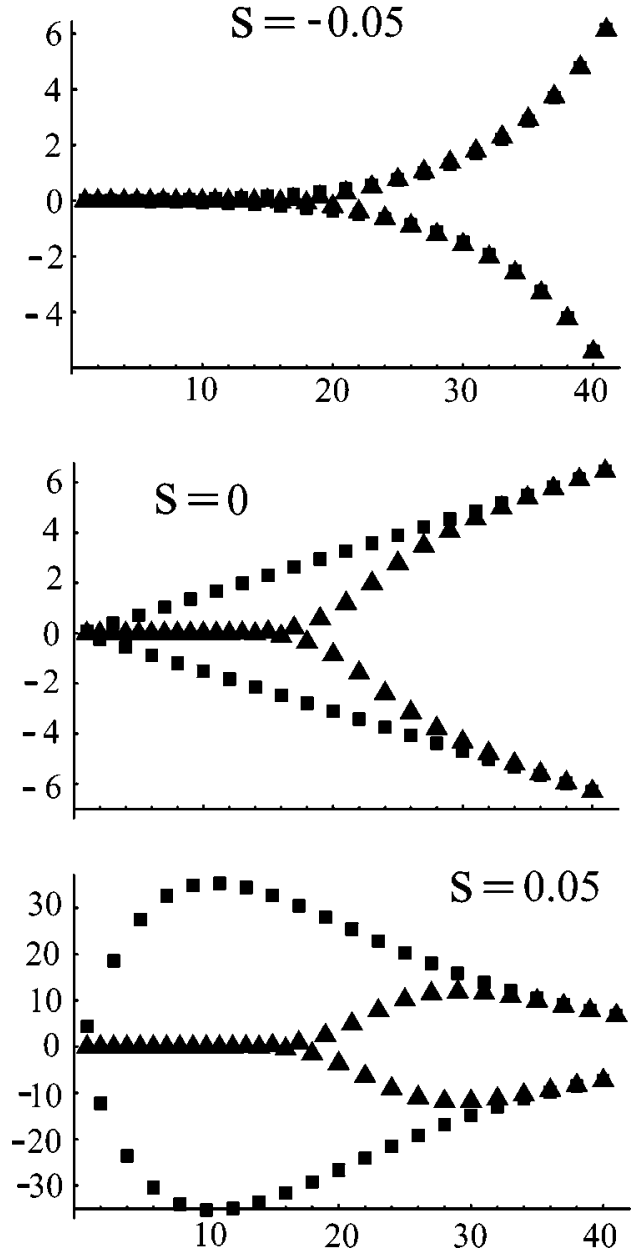

FIG. 1. Spectrum of $\Delta^{(N)}(\Omega ; s)$ (squares) and $\Lambda^{(j)}(\Omega ; s)$ (triangles) for $2 j=N=40$ and $s=0, \pm 0.05$.

The marginal distributions (2.19) inherit the good transformation properties of the quadrature distributions (2.7) under $\mathrm{SU}(2)$ transformations since

$$
W_{U \rho U^{\dagger}}(\boldsymbol{\Omega} ; s)=W_{\rho}\left(\mathcal{V}^{t} \boldsymbol{\Omega} ; s\right),
$$

where $\mathcal{V}^{t}$ is the transpose of the matrix $\mathcal{V}$ defined by the relation

$$
U^{\dagger} S U=\mathcal{V} \boldsymbol{S} .
$$

This transformation law is also satisfied by the $\mathrm{SU}(2)$ distributions [1-3].

There is another property that is not inherited. This is the proportionality between phase-space averages and Hilbertspace traces. For quadrature distributions it holds that [8]

$$
\pi^{2} \int d^{2} \alpha_{1} d^{2} \alpha_{2} W_{A}\left(\alpha_{1}, \alpha_{2} ;-s\right) W_{B}\left(\alpha_{1}, \alpha_{2} ; s\right)=\operatorname{tr}(A B),
$$

and equivalently for the $\mathrm{SU}(2)$ distributions [3],

$$
\frac{4 \pi}{2 j+1} \int d \Omega \mathcal{W}_{A}(\Omega ;-s) \mathcal{W}_{B}(\Omega ; s)=\operatorname{tr}(A B),
$$

which are valid for every $s$. However, this no longer holds literally for the polarization marginals. There are two consequences that can be derived from this fact. On the one hand, it is possible to restore this property by introducing another operator-function correspondence $\widetilde{W}_{A} \leftrightarrow A$ such that

$$
\int d \Omega \tilde{W}_{A}(\Omega ;-s) W_{B}(\Omega ; s)=\operatorname{tr}(A B) .
$$

This possibility is developed in Sec. IV. On the other hand, there is the possibility of using the marginal Wigner function to measure the degree of polarization of quantum states, as discussed in Sec. VI.

\section{DUAL DISTRIBUTIONS}

The strategy addressed in this work actually defines two phase-space formalisms. In addition to the correspondence defined above, $W(\Omega ; s)=\operatorname{tr}[A \Delta(\Omega ; s)]$ we can devise a dual definition $\widetilde{W}(\Omega ; s)$ that emerges when expressing every observable $A$ commuting with $S_{0}$ as an expansion of the form

$$
A=\int d \Omega \tilde{W}(\Omega ;-s) \Delta(\Omega ; s) .
$$

Assuming a linear relationship between operators and functions,

$$
\widetilde{W}(\Omega ; s)=\operatorname{tr}[A \widetilde{\Delta}(\Omega ; s)],
$$

we have that the dual operators $\widetilde{\Delta}(\Omega ; s)$ should be determined by the consistency condition

$$
A=\int d \Omega \operatorname{tr}[A \tilde{\Delta}(\Omega ;-s)] \Delta(\Omega ; s),
$$

leading to

$$
\int d \Omega\langle j, p|\widetilde{\Delta}(\Omega ;-s)| j, m\rangle\langle j, k|\Delta(\Omega ; s)| j, q\rangle=\delta_{p, q} \delta_{m, k} .
$$

If these equations can be solved, then Eq. (4.1) holds for every $A$ commuting with $S_{0}$. Moreover, given the explicit symmetry in Eq. (4.4) between $\Delta$ and $\widetilde{\Delta}$, the fulfillment of Eq. (4.4) also allows us to express $A$ in the form

$$
A=\int d \Omega W(\Omega ;-s) \widetilde{\Delta}(\Omega ; s) .
$$

In the next section we will solve Eqs. (4.4) for $j=1 / 2$ and $j=1$.

For quadrature and $\mathrm{SU}(2)$ distributions the direct and dual definitions coincide: $\widetilde{T}(\alpha ; s)=T(\alpha ; s)$ [8] and $\tilde{\Lambda}(\Omega ; s)$ $=\Lambda(\Omega ; s)[3]$. However, we have found no general relation of this kind between $\widetilde{\Delta}(\Omega ; s)$ and $\Delta(\Omega ; s)$ (leaving aside the simplest two-dimensional case $j=N / 2=1 / 2$ examined below). 
The fulfillment of Eq. (4.4) restores the relation between phase-space averages and Hilbert-space traces-for example, in the form

$$
\begin{aligned}
\operatorname{tr}(A B)= & \int d \Omega W_{A}(\Omega ;-s) \tilde{W}_{B}(\Omega ; s)=\int d \Omega W_{B}(\Omega ; \\
& -s) \tilde{W}_{A}(\Omega ; s) .
\end{aligned}
$$

Moreover, it also allows us to establish a link between the distributions with different values of $s$. By using Eq. (4.5) for $A=\rho$ and $s=-s^{\prime}$ we get

$$
W(\Omega ; s)=\operatorname{tr}[\rho \Delta(\Omega ; s)]=\int d \Omega^{\prime} K\left(\Omega, \Omega^{\prime} ; s, s^{\prime}\right) W\left(\Omega^{\prime} ; s^{\prime}\right),
$$

with

$$
K\left(\Omega, \Omega^{\prime} ; s, s^{\prime}\right)=\operatorname{tr}\left[\Delta(\Omega ; s) \widetilde{\Delta}\left(\Omega^{\prime} ;-s^{\prime}\right)\right] .
$$

This is the angular analog of the convolution relation between quadrature distributions [8]. A similar relation holds for the $\mathrm{SU}(2)$ distributions $[3,4]$.

\section{EXAMPLES}

Next we illustrate the results of the previous sections by particularizing them to $\mathrm{SU}(2)$ coherent states, number states, and one-photon and two-photon states.

\section{A. $\mathrm{SU}(2)$ coherent states}

For SU(2) coherent states we can take advantage of the $\mathrm{SU}(2)$ covariance by considering without loss of generality the case $|n\rangle_{1}|0\rangle_{2}$ which corresponds to a SU(2) coherent state centered at the north pole of the Poincaré sphere. The evaluation of the distribution simplifies if we express Eq. (2.19) in the form

$$
\begin{aligned}
\Delta(\Omega ; s)= & \frac{1}{4 \pi}\left(\frac{s+1}{s-1}\right)^{a_{2}^{\dagger}(\Omega) a_{2}(\Omega)}\left(1+\frac{2}{1-s} a_{1}^{\dagger}(\Omega) a_{1}(\Omega)\right) \\
= & \frac{1}{4 \pi}\left(\frac{s+1}{s-1}\right)^{a_{2}^{\dagger}(\Omega) a_{2}(\Omega)}+\frac{1}{4 \pi} \frac{2}{1-s} a_{1}^{\dagger}(\Omega) \\
& \times\left(\frac{s+1}{s-1}\right)^{a_{2}^{\dagger}(\Omega) a_{2}(\Omega)} a_{1}(\Omega),
\end{aligned}
$$

where $a_{1}(\Omega)=U a_{1} U^{\dagger}$ and $a_{2}(\Omega)=U a_{2} U^{\dagger}$ are given by Eq. (2.18). The mean value of $\Delta(\Omega ; s)$ in the state $|n\rangle_{1}|0\rangle_{2}$ can be easily evaluated taking into account that mode $a_{2}$ is in vacuum and using the general relation

$$
\nu^{a^{\dagger} a}=: e^{(\nu-1) a^{\dagger} a}:,
$$

where : : denotes normal order.

This leads to

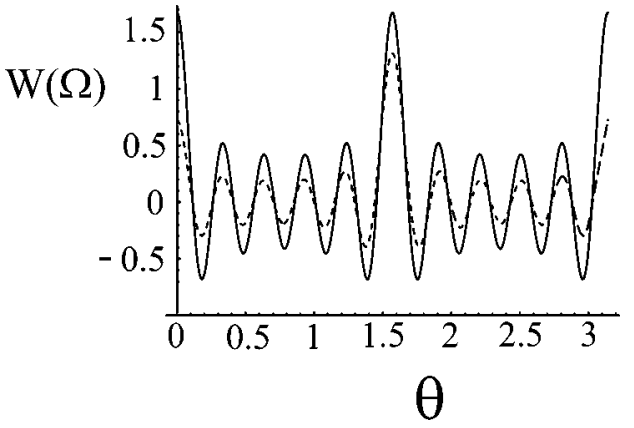

FIG. 2. Plot of the Wigner functions $(s=0) W(\Omega)$ (solid line) and $\mathcal{W}(\Omega)$ (dotted line), as functions of $\theta$ for the number state $|10\rangle_{1}|10\rangle_{2}$.

$$
\begin{aligned}
W(\Omega ; s)= & \frac{1}{4 \pi}\left(1+\frac{2}{s-1} \sin ^{2} \frac{\theta}{2}\right)^{n-1} \\
& \times\left(1-\frac{2 n}{s-1}+2 \frac{n+1}{s-1} \sin ^{2} \frac{\theta}{2}\right) .
\end{aligned}
$$

On the other hand, the expression for the $\mathrm{SU}(2)$ distribution is

$$
\mathcal{W}(\Omega ; s)=\frac{1}{4 \pi} \sum_{\ell=0}^{n}(2 \ell+1)\langle n / 2, n / 2 ; \ell, 0 \mid n / 2, n / 2\rangle^{1-s} P_{\ell}(\cos \theta),
$$

where $P_{\ell}$ are the Legendre polynomials.

In spite of the fact that these expressions are seemingly very different, for $s=0$ they are practically indistinguishable even for small photon numbers. The particular examples $n$ $=1,2$ are examined below.

\section{B. Number states}

For general number states $\left|n_{1}\right\rangle_{1}\left|n_{2}\right\rangle_{2}$ the expression for the $\mathrm{SU}(2)$ distribution is

$$
\mathcal{W}(\Omega ; s)=\frac{1}{4 \pi} \sum_{\ell=0}^{2 j}(2 \ell+1) \frac{\langle j, m ; \ell, 0 \mid j, m\rangle}{\langle j, j ; \ell, 0 \mid j, j\rangle} P_{\ell}(\cos \theta),
$$

being

$$
j=\frac{n_{1}+n_{2}}{2}, \quad m=\frac{n_{1}-n_{2}}{2} .
$$

Unfortunately we have found no simple closed expression for the marginal distributions when $n_{1} n_{2} \neq 0$. Nevertheless, numerical calculations are simple. In Fig. 2 we represent the $\mathrm{SU}(2)$ (dotted line) and marginal (solid line) Wigner functions $(s=0)$ for the number state $|10\rangle_{1}|10\rangle_{2}$. It can be appreciated that both distributions are very similar.

\section{One-photon states}

The subspace with just one photon shared by two field modes is spanned by the number states 


$$
|1\rangle_{1}|0\rangle_{2}=\left(\begin{array}{l}
1 \\
0
\end{array}\right), \quad|0\rangle_{1}|1\rangle_{2}=\left(\begin{array}{l}
0 \\
1
\end{array}\right) .
$$

In this basis we get

$$
\begin{aligned}
& \Delta(\Omega ; s)=\frac{1}{4 \pi}\left(1+\frac{2}{1-s} \boldsymbol{\Omega} \cdot \boldsymbol{\sigma}\right), \\
& \Lambda(\Omega ; s)=\frac{1}{4 \pi}\left(1+\sqrt{3^{s+1}} \boldsymbol{\Omega} \cdot \boldsymbol{\sigma}\right), \\
& \widetilde{\Delta}(\Omega ; s)=\frac{1}{2}\left(1+3 \frac{s+1}{2} \boldsymbol{\Omega} \cdot \boldsymbol{\sigma}\right),
\end{aligned}
$$

where $\sigma$ are the three Pauli matrices.

Every one-photon density matrix can be expressed as

$$
\rho=\frac{1}{2}(1+\boldsymbol{v} \cdot \boldsymbol{\sigma}),
$$

where $\boldsymbol{v}$ is a real vector with $\boldsymbol{v} \leqslant 1$. For $|\boldsymbol{v}|=1$ we get pure states that are all them $\mathrm{SU}(2)$ coherent states. The associated polarization distributions are

$$
\begin{gathered}
W(\Omega ; s)=\frac{1}{4 \pi}\left(1+\frac{2}{1-s} \boldsymbol{\Omega} \cdot \boldsymbol{v}\right), \\
\mathcal{W}(\Omega ; s)=\frac{1}{4 \pi}\left(1+\sqrt{3^{1+s} \boldsymbol{\Omega} \cdot \boldsymbol{v}}\right), \\
\tilde{W}(\Omega ; s)=\frac{1}{4 \pi}\left(1+3 \frac{s+1}{2} \boldsymbol{\Omega} \cdot \boldsymbol{v}\right),
\end{gathered}
$$

and in this last expression we have normalized $\tilde{W}(\Omega ; s)$ so that

$$
\int d \Omega \tilde{W}(\Omega ; s)=1 .
$$

In this particular case there is a simple relation between the marginal and the $\mathrm{SU}(2)$ distributions in the form

$$
\Delta(\Omega ; s)=\Lambda\left(\Omega ; s^{\prime}\right), \quad s^{\prime}=\frac{2}{\ln 3} \ln \frac{2}{1-s}-1 .
$$

Furthermore, there is also a simple relation between marginal and dual distributions:

$$
\widetilde{\Delta}(\Omega ; s)=2 \pi \Delta\left(\Omega ; s^{\prime}\right), \quad s^{\prime}=1-\frac{4}{3(1+s)} .
$$

\section{Two-photon states}

The subspace with two photons shared by two field modes is spanned by the number states

$$
|1\rangle=|2\rangle_{1}|0\rangle_{2}, \quad|0\rangle=|1\rangle_{1}|1\rangle_{2}, \quad|-1\rangle=|0\rangle_{1}|2\rangle_{2} .
$$

In this basis we get the following matrix elements for the marginal phase-space point operators $\Delta(\Omega ; s)$ :

$$
\begin{aligned}
&\langle \pm 1|\Delta(\Omega ; s)| \pm 1\rangle= \frac{1}{4 \pi(s-1)^{2}}[s(s-2) \mp 2(2 s-1) \cos \theta \\
&\left.+3 \cos ^{2} \theta\right], \\
&\langle 0|\Delta(\Omega ; s)| 0\rangle= \frac{1}{4 \pi(s-1)^{2}}\left[s(s-2)+3-6 \cos ^{2} \theta\right], \\
&\langle \pm 1|\Delta(\Omega ; s)| 0\rangle=\frac{1}{2 \sqrt{2} \pi(s-1)^{2}}(1-2 s \pm 3 \cos \theta) \sin \theta e^{\mp i \phi}, \\
&\langle 1|\Delta(\Omega ; s)|-1\rangle=\frac{3}{4 \pi(s-1)^{2}} \sin ^{2} \theta e^{-i 2 \phi} .
\end{aligned}
$$

For the $\mathrm{SU}(2)$ operators $\Lambda(\Omega ; s)$ we have

$$
\begin{gathered}
\langle \pm 1|\Lambda(\Omega ; s)| \pm 1\rangle=\frac{1}{8 \sqrt{2} \pi}\left[2 \sqrt{2}-\sqrt{2^{s} 5^{(s+1)}} \pm 6 \sqrt{2^{s}} \cos \theta\right. \\
\left.+3 \sqrt{2^{s 5^{s+1}}} \cos ^{2} \theta\right], \\
\langle 0|\Lambda(\Omega ; s)| 0\rangle=\frac{1}{4 \pi}\left[1+\sqrt{2^{s-1} 5^{s+1}}-3 \sqrt{2^{s-1} 5^{s+1}} \cos ^{2} \theta\right], \\
\langle \pm 1|\Lambda(\Omega ; s)| 0\rangle=\frac{3 \sqrt{2^{s}}}{8 \pi}\left(1 \pm \sqrt{5^{s+1}} \cos \theta\right) \sin \theta e^{\mp i \phi}, \\
\langle 1|\Lambda(\Omega ; s)|-1\rangle=\frac{3 \sqrt{2^{s-1} 5^{s+1}}}{8 \pi} \sin ^{2} \theta e^{-i 2 \phi} .
\end{gathered}
$$

Concerning the dual distributions we have been able to obtain the solution of Eq. (4.4) as

$$
\begin{gathered}
\langle \pm 1|\widetilde{\Delta}(\Omega ; s)| \pm 1\rangle=\frac{5}{8}(s+1)^{2}\left[\frac{3-5 s(s+2)}{15(s+1)^{2}}\right. \\
\left. \pm \frac{6}{5(2 s+1)} \cos \theta+\cos ^{2} \theta\right], \\
\langle 0|\widetilde{\Delta}(\Omega ; s)| 0\rangle=\frac{1}{12}\left[9+5 s(s+2)-15(s+1)^{2} \cos ^{2} \theta\right], \\
\langle \pm 1|\widetilde{\Delta}(\Omega ; s)| 0\rangle=\frac{5}{4 \sqrt{2}}(s+1)^{2}\left[\frac{3}{5(2 s+1)} \pm \cos \theta\right] \sin \theta e^{\mp i \phi}, \\
\langle 1|\tilde{\Delta}(\Omega ; s)|-1\rangle=\frac{5}{8}(s+1)^{2} \sin ^{2} \theta e^{-2 i \phi} .
\end{gathered}
$$

Let us particularize to the Wigner function $s=0$ for the number state $|2\rangle_{1}|0\rangle_{2}$ which is a $\mathbf{S U}(2)$ coherent state,

$$
W(\Omega ; 0)=\frac{1}{4 \pi}\left(2 \cos \theta+3 \cos ^{2} \theta\right),
$$

$$
\mathcal{W}(\Omega ; 0)=\frac{1}{32 \pi}\left(8-2 \sqrt{10}+12 \sqrt{2} \cos \theta+6 \sqrt{10} \cos ^{2} \theta\right),
$$




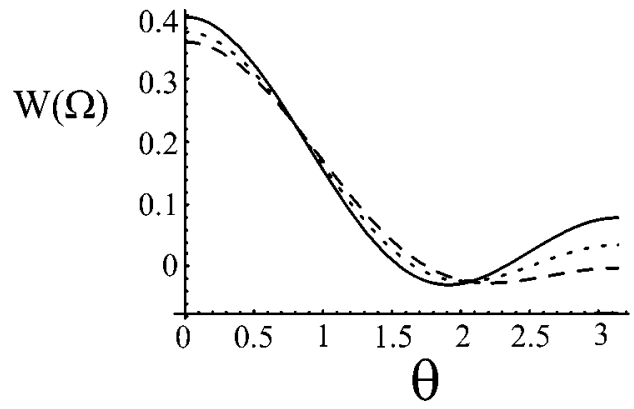

FIG. 3. Plot of the Wigner functions $(s=0) W(\Omega ; 0)$ (solid line), $\widetilde{W}(\Omega ; 0)$ (dashed line), and $\mathcal{W}(\Omega ; 0)$ (dotted line), as functions of $\theta$ for the state $|2\rangle_{1}|0\rangle_{2}$.

$$
\tilde{W}(\Omega ; 0)=\frac{3}{32 \pi}\left(1+6 \cos \theta+5 \cos ^{2} \theta\right),
$$

where we have normalized $\tilde{W}(\Omega ; 0)$ as in Eq. (5.11). We have plotted these functions in Fig. 3 as functions of $\theta$.

On the other hand, for the state $|1\rangle_{1}|1\rangle_{2}$, which is a $\mathrm{SU}(2)$ squeezed state, $[9,18,19]$,

$$
\begin{gathered}
W(\Omega ; 0)=\frac{3}{4 \pi}\left(1-2 \cos ^{2} \theta\right), \\
\mathcal{W}(\Omega ; 0)=\frac{1}{8 \pi}\left(2+\sqrt{10}-3 \sqrt{10} \cos ^{2} \theta\right), \\
\tilde{W}(\Omega ; 0)=\frac{3}{16 \pi}\left(3-5 \cos ^{2} \theta\right),
\end{gathered}
$$

where we have normalized $\tilde{W}(\Omega ; 0)$ as in Eq. (5.11). We have plotted these functions in Fig. 4 as functions of $\theta$. Note that in both examples $\mathcal{W}(\Omega ; 0)$ is intermediate between $W(\Omega ; 0)$ and $\tilde{W}(\Omega ; 0)$.

\section{DEGREE OF POLARIZATION}

In classical optics the degree of polarization is measured in terms of the Stokes parameters, being proportional to $|\langle\boldsymbol{S}\rangle|$. However, there are many situations where this does not reflect the polarization properties of the quantum states of

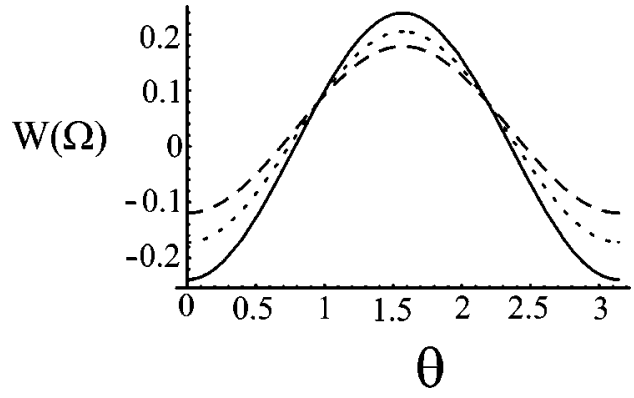

FIG. 4. Plot of the Wigner functions $(s=0) W(\Omega ; 0)$ (solid line), $\widetilde{W}(\Omega ; 0)$ (dashed line), and $\mathcal{W}(\Omega ; 0)$ (dotted line), as functions of $\theta$ for the state $|1\rangle_{1}|1\rangle_{2}$.

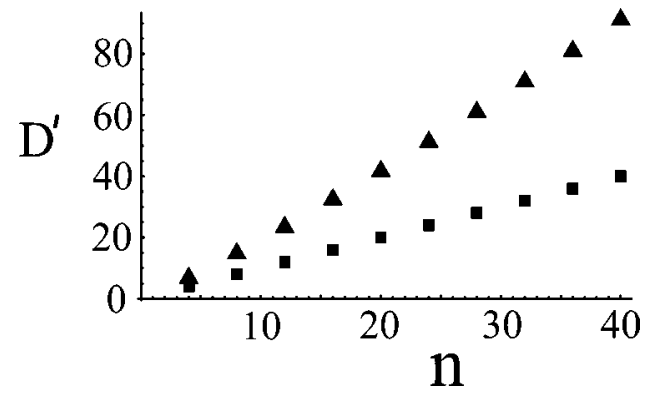

FIG. 5. Plot of the degree of polarization, $D^{\prime}$, for the $\mathrm{SU}(2)$ coherent states $|n\rangle_{1}|0\rangle_{2}$ (squares) and the number states $|n / 2\rangle_{1}|n / 2\rangle_{2}$ (triangles) as functions of the number of photons, $n$.

light. For example, there are many quantum states for which $\langle\boldsymbol{S}\rangle=0$ that are not unpolarized. This is the case of the number states $|n\rangle_{1}|n\rangle_{2}$. Moreover, the classical degree of polarization depends only on second-order correlations of the field amplitudes. However, in quantum optics higher-order correlations can be crucial. This is the case of polarization squeezing, which is defined in terms of the fluctuations of the Stokes operators instead of their mean values $[9,18,19]$.

To avoid these shortcomings of the classic definition we have recently introduced a measure of polarization as the distance between the polarization distribution and the uniform distribution associated with fully unpolarized light, $W_{\text {unpol }}(\Omega ; s)=1 /(4 \pi)[9]$.

As a suitable polarization distribution we have considered the marginal $Q$ function [which is the only case that coincides with the corresponding $\mathrm{SU}(2)$ distribution]. We can measure the distance to fully unpolarized light in the form

$D=4 \pi \int d \Omega\left[W(\Omega ;-1)-\frac{1}{4 \pi}\right]^{2}=4 \pi \int d \Omega W^{2}(\Omega ;-1)-1$.

In principle we might have considered another distribution, such as the $\mathrm{SU}(2)$ Wigner function $\mathcal{W}(\Omega ; 0)$, for example. However, this is not a suitable choice since, because of property (3.12), the integration of the square of the Wigner function only depends on the trace of the square of the density matrix, so this is just a measure of purity rather than a measure of polarization. For example, it would take exactly the same value for all pure states, so it can hardly be related to polarization properties.

In this work we have found another polarization Wigner function $W(\Omega ; 0)$ that does not encounter this difficulty, as discussed at the end of Sec. III. In principle, this distribution may be used in order to assess the degree of polarization in the form

$$
D^{\prime}=4 \pi \int d \Omega\left[W(\Omega ; 0)-\frac{1}{4 \pi}\right]^{2}=4 \pi \int d \Omega W^{2}(\Omega ; 0)-1 .
$$

Next we examine this possibility.

In Fig. 5 we represent $D^{\prime}$ for $\mathrm{SU}(2)$ coherent states $|n\rangle_{1}|0\rangle_{2}$ (squares) and number states $|n / 2\rangle_{1}|n / 2\rangle_{2}$ (triangles) as functions of the total number of photons $n$. 


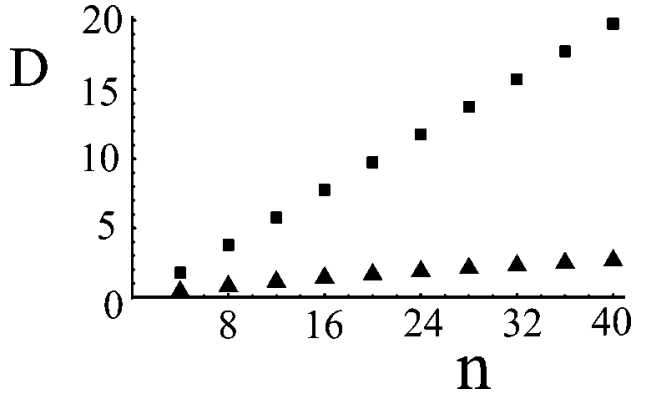

FIG. 6. Plot of the degree of polarization, $D$, for the $\mathrm{SU}(2)$ coherent states $|n\rangle_{1}|0\rangle_{2}$ (squares) and the number states $|n / 2\rangle_{1}|n / 2\rangle_{2}$ (triangles) as functions of the number of photons, $n$.

It can be appreciated that the degree of polarization increases almost linearly with $n$ in both cases. The rate of increase of $D^{\prime}$ is faster for the number states $|n / 2\rangle_{1}|n / 2\rangle_{2}$. This contradicts the result obtained when using the $Q$ function which predicts

$$
D_{n, 0}=\frac{n^{2}}{2 n+1}, \quad D_{n / 2, n / 2}=\frac{(n+1)^{2} n !^{4}}{(2 n+1) !(n / 2) !^{4}}-1 .
$$

These functions are represented in Fig. 6 where it can be appreciated that the degree of polarization, $D$, increases faster for the $\mathrm{SU}(2)$ coherent states $|n\rangle_{1}|0\rangle_{2}$.

We think that there are reasons supporting the conjecture that the definition based on the $Q$ function provides the right result. The equivalence between phase-space averages and Hilbert-space traces satisfied by the quadrature Wigner function imposes the condition that most states must have Wigner functions with negative values (since the phase-space average of the product of orthogonal states must vanish). Since the distributions are always normalized,

$$
\int d \Omega W(\Omega ;-1)=\int d \Omega W(\Omega ; 0)=1,
$$

the negative values of $W(\Omega ; 0)$ must be compensated for by larger positive values in order to preserve the normalization. This implies strong oscillations that move the distribution away from the unpolarized constant value $1 /(4 \pi)$. This increases the degree of polarization. On the other hand, the $Q$ function does not experience this enlarged oscillation since it remains always positive.

In Fig. 7 we represent $W(\Omega ;-1)$ and $W(\Omega ; 0)$ for the state $|n / 2\rangle_{1}|n / 2\rangle_{2}$ with $n=20$ photons. This plot illustrates the negative values and strong oscillations of the Wigner function in comparison with the $Q$ function.

Therefore, the structural properties of the Wigner functions would artificially increase the degree of polarization of

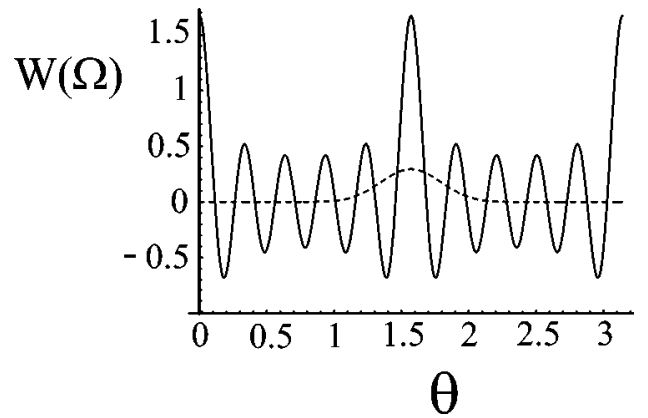

FIG. 7. Plot of $W(\Omega ;-1)$ (dashed line) and $W(\Omega ; 0)$ (solid line) for the state $|n / 2\rangle_{1}|n / 2\rangle_{2}$ with $n=20$ photons as functions of $\theta$.

states with nonpositive Wigner functions. Therefore, we think that the degree of polarization is better measured by using the $Q$ function since it is the only distribution that is non-negative for all states, being closer to the usual understanding of polarization distribution.

\section{CONCLUSIONS}

We have provided a route to define quantum phase-space formalisms for polarization on the Poincaré sphere, as suitable marginals of $s$-ordered operator-function correspondences for quadrature variables.

We have shown that this procedure defines polarization distributions that are different from the approaches introduce so far (leaving aside the case of the $Q$ function). Nevertheless, we have found that for all the cases examined the marginal Wigner distribution is very similar to the $\mathrm{SU}(2)$ Wigner function.

The results obtained in this work can have interesting experimental consequences. On the one hand, the marginals derived from $s$-ordered distributions provide simple accessible experimental procedures for determining polarization properties. For example, this includes recently introduced measures of degree of polarization and polarization correlations $[9,10]$. In particular, polarization correlations provide a fundamental example of experimentally generated entanglement with applications in rapidly developing areas such as quantum computation and cryptography [11]. Another practical consequence of this approach to be elaborated elsewhere is the study and detection of nonclassical properties of field states, since they are generally expressed as negative values for $s$-ordered distributions [17].

\section{ACKNOWLEDGMENT}

This work has been supported by Project No. FIS200401814 of the Spanish Dirección General de Investigación del Ministerio de Educación y Ciencia. 
[1] G. S. Agarwal, Phys. Rev. A 24, 2889 (1981); J. P. Dowling, G. S. Agarwal, and W. P. Schleich, ibid. 49, 4101 (1994).

[2] J. C. Várilly and J. M. Gracia-Bondía, Ann. Phys. (N.Y.) 190, 107 (1989).

[3] C. Brif and A. Mann, J. Phys. A 31, L9 (1998); Phys. Rev. A 59, 971 (1999); J. Opt. B: Quantum Semiclassical Opt. 2, 245 (2000).

[4] M. P. Miranda, F. J. Aoiz, L. Bañares, and V. Sáez Rábanos, J. Chem. Phys. 111, 5368 (1999); M. P. Miranda and F. J. Aoiz, Phys. Rev. Lett. 93, 083201 (2004).

[5] F. T. Arecchi, E. Courtens, R. Gilmore, and H. Thomas, Phys. Rev. A 6, 2211 (1972).

[6] D. M. Kaplan and G. C. Summerfield, Phys. Rev. 187, 639 (1969); M. O. Scully, Phys. Rev. D 28, 2477 (1983); G. Ramachandran, A. R. Usha-Devi, P. Devi, and S. Sirsi, Found. Phys. 26, 401 (1996); S. M. Chumakov, A. Frank, and K. B. Wolf, Phys. Rev. A 60, 1817 (1999); V. P. Karassiov and A. V. Masalov, J. Opt. B: Quantum Semiclassical Opt. 4, S366 (2002); S. M. Chumakov, A. B. Klimov, and K. B. Wolf, Phys. Rev. A 61, 034101 (2001).

[7] W. K. Wootters, Ann. Phys. (N.Y.) 176, 1 (1987); O. Cohendet, Ph. Combe, M. Sirugue, and M. Sirugue-Collin, J. Phys. A 21, 2875 (1988); D. Galetti and A. F. R. Toledo Piza, Physica A 149, 267 (1988); J. A. Vaccaro and D. T. Pegg, Phys. Rev. A 41, 5156 (1990); A. Lukš and V. Peřinová, Quantum Opt. 6, 125 (1994); U. Leonhardt, Phys. Rev. Lett. 74, 4101 (1995); Phys. Rev. A 53, 2998 (1996); T. Opatrný, D.-G. Welsch, and B. Bužek, ibid. 53, 3822 (1996); C. Miquel, J. P. Paz, and M. Saraceno, ibid. 65, 062309 (2002); J. P. Paz, ibid. 65, 062311 (2002); A. Luis and J. Peřina, J. Phys. A 31, 1423 (1998).

[8] K. E. Cahill and R. J. Glauber, Phys. Rev. 177, 1857 (1969); 177, 1882 (1969); M. Hillery, R. F. O’Connell, M. O. Scully, and E. P. Wigner, Phys. Rep. 106, 121 (1984).

[9] A. Luis, Phys. Rev. A 66, 013806 (2002).

[10] A. Luis, Opt. Commun. 216, 165 (2003); Phys. Rev. A 69, 023803 (2004).

[11] The Physics of Quantum Information, edited by D. Bouwmeester, A. Ekert, and A. Zeilinger (Springer, Berlin, 2001); H. Paul, Introduction to Quantum Optics (Cambridge University Press, Cambridge, England, 2004).

[12] W. Schleich, R. J. Horowicz, and S. Varro, Phys. Rev. A 40, 7405 (1989); S. L. Braunstein and C. M. Caves, ibid. 42, 4115 (1990); R. Tanaś, A. Miranowicz, and Ts. Gantsog, Phys. Scr. T48, 53 (1993); U. Leonhardt, J. A. Vaccaro, B. Böhmer, and H. Paul, Phys. Rev. A 51, 84 (1995); A. Alexandrescu and D. H. Schiller, J. Opt. B: Quantum Semiclassical Opt. 6, 360 (2004).

[13] Ch. Brosseau, Fundamentals of Polarized Light: A Statistical Optics Approach (Wiley, New York, 1998).

[14] G. García Calderón and M. Moshinsky, J. Phys. A 13, L185
(1980); R. Dirl, P. Kasperkovitz, and M. Moshinsky, ibid. 21, 1835 (1988); A. K. Ekert and P. L. Knight, Phys. Rev. A 43, 3934 (1991); L. Knöll, S. Scheel, E. Schmidt, D.-G. Welsch, and A. V. Chizhov, ibid. 59, 4716 (1999); S. Scheel, L. Knöll, T. Opatrný, and D.-G. Welsch, ibid. 62, 043803 (2000); A. Luis, ibid. 70, 052118 (2004).

[15] U. Leonhardt and H. Paul, Prog. Quantum Electron. 19, 89 (1995); U. Leonhardt, Measuring the Quantum State of light (Cambridge University Press, Cambridge, England, 1997); D.-G. Welsch, W. Vogel, and T. Opatrný, in Progress in Optics, edited by E. Wolf (Elsevier Science, Amsterdam, 1999), Vol. 39; N. G. Walker and J. E. Carroll, Electron. Lett. 20, 981 (1984); Opt. Quantum Electron. 18, 355 (1986); K. Vogel and H. Risken, Phys. Rev. A 40, 2847 (1989); D. T. Smithey, M. Beck, M. G. Raymer, and A. Faridani, Phys. Rev. Lett. 70, 1244 (1993); J. R. Torgerson and L. Mandel, ibid. 76, 3939 (1996); S. Schiller, G. Breitenbach, S. F. Pereira, T. Müller, and J. Mlynek, ibid. 77, 2933 (1996); G. Breitenbach, S. Schiller, and J. Mlynek, Nature (London) 387, 471 (1997); M. Beck, C. Dorrer, and I. A. Walmsley, Phys. Rev. Lett. 87, 253601 (2001); A. I. Lvovsky, H. Hansen, T. Aichele, O. Benson, J. Mlynek, and S. Schiller, ibid. 87, 050402 (2001); P. Bertet, A. Auffeves, P. Maioli, S. Osnaghi, T. Meunier, M. Brune, J. M. Raimond, and S. Haroche, ibid. 89, 200402 (2002); A. I. Lvovsky and S. A. Babichev, Phys. Rev. A 66, 011801(R) (2002).

[16] W. Band and J. L. Park, Found. Phys. 1, 133 (1970); J. L. Park and W. Band, ibid. 1, 211 (1971); W. Band and J. L. Park, ibid. 1, 339 (1970); R. G. Newton and B.-L. Young, Ann. Phys. (N.Y.) 49, 393 (1968); G. S. Agarwal, Phys. Rev. A 57, 671 (1998); J. P. Amiet and S. Weigert, J. Opt. B: Quantum Semiclassical Opt. 2, 118 (2000); A. B. Klimov, O. V. Man'ko, V. I. Man'ko, Y. F. Smirnov, and V. N. Tolstoy, J. Phys. A 35, 6101 (2002); S. Weigert, J. Opt. B: Quantum Semiclassical Opt. 6, 489 (2004); H. F. Hofmann and S. Takeuchi, Phys. Rev. A 69, 042108 (2004).

[17] R. J. Glauber, Phys. Rev. 131, 2766 (1963); U. M. Titulaer and R. J. Glauber, Phys. Rev. 140, B676 (1965); M. Hillery, Phys. Lett. 111A, 409 (1985); C. T. Lee, Phys. Rev. A 44, R2775 (1991); 52, 3374 (1995); N. Lükenhaus and S. M. Barnett, ibid. 51, 3340 (1995); A. F. de Lima and B. Baseia, ibid. 54, 4589 (1996); J. Janszky, M. G. Kim, and M. S. Kim, ibid. 53, 502 (1996); J. M. C. Malbouisson and B. Baseia, e-print quantph/0209107; V. V. Dodonov, J. Opt. B: Quantum Semiclassical Opt. 4, R1 (2002).

[18] M. Kitagawa and M. Ueda, Phys. Rev. A 47, 5138 (1993); D. J. Wineland, J. J. Bollinger, W. M. Itano, and D. J. Heinzen, ibid. 50, 67 (1994).

[19] A. Luis and L. L. Sánchez-Soto, in Progress in Optics, edited by E. Wolf (Elsevier, Amsterdam, 2000), Vol. 41, p. 421. 